\title{
Inflammatory Breast Cancer - Does the Confirmation of Dermal Lymphatic Invasion Predict the Worst Outcome?
}

\author{
Zorica Tomasevic ${ }^{1}$ and Daniela Kolarevic ${ }^{2}$
}

1. Director, Daily Chemotherapy Hospital, Institute for Oncology and Radiology of Serbia;

2. Oncologist, Daily Chemotherapy Hospital, Institute for Oncology and Radiology of Serbia

\begin{abstract}
Inflammatory breast carcinoma (IBC) is a rare but aggressive breast cancer $(\mathrm{BC})$ subtype. General prognosis is poor with higher incidence of axillary node involvement and metastatic disease at presentation $(\sim 30 \%)$ as compared with common BC $(\sim 10 \%)$. The typical IBC is characterised by rapid onset of localised or generalised skin redness, involving at least one-third of the breast, warmth and usually presence of skin oedema (peau d'orange), often without an underlying tumour mass. This clinical presentation of skin inflammation is a consequence of pathological plugging of the dermal lymphatic of the breast with tumour emboli. Dermal lymphatic invasion (DLI), although not mandatory for IBC diagnosis, is one of the hallmarks of this malignancy and can be proven in up to $75-80 \%$ of IBCs. Surprisingly, there are very few data regarding whether the presence of DLI independently contributes to IBC prognosis as compared with IBC without DLI.
\end{abstract}

\section{Keywords}

Inflammatory breast carcinoma (IBC), dermal lymphatic invasion (DLI), prognosis of IBC, IBC molecular characteristics, IBC subtypes

Disclosure: The authors have no conflicts of interest to declare.

Received: 4 December 2011 Accepted: 16 December 2011 Citation: European Oncology \& Haematology, 2012;8(1):20-3 DOI: 10.17925/EOH.2012.08.01.20

Correspondence: Zorica Tomasevic, Institute for Oncology and Radiology of Serbia, 11000 Belgrade, Serbia. E: zoricant@ncrc.ac.rs

Breast carcinoma (BC) is one of the commonest cancers in the world, and definitely the most common malignant disease in women with more than one million newly diagnosed patients each year.

However, among this common, but heterogeneous, malignancy, there is a subset of rare $\mathrm{BC}$ form called inflammatory breast carcinoma (IBC), representing approximately $1-5 \%$ of all breast cancer types. ${ }^{1}$ The term inflammatory was first proposed by Lee and Tannenbaum in 1924, and this is still the most appropriate name for this form of breast carcinoma because it actually describes very accurately the typical clinical presentation. ${ }^{2}$

\section{Clinical Presentation and Diagnosis}

The hallmark of this rare BC type is rapid onset of localised or generalised typical skin redness, involving at least one-third of the breast, breast warmth and usually typical oedema (peau d'orange), often without an underlying tumour mass.

Although this specific form of BC was first recognised long ago, diagnostic criteria were inconsistent and IBC had been often grouped with locally advanced breast cancer ( $L A B C)$, especially if accompanied with secondary inflammation (see Figure 1). This inconsistency was leading to a discrepancy in incidence reporting, especially in some parts of the world. ${ }^{2}$ For instance, the first reports about rapidly developing $\mathrm{BC}$ from Tunisia stated high IBC incidence exceeding $50 \%$ of patients with $\mathrm{BC}$, provoking speculation that some unidentified environmental factors could contribute to IBC development. ${ }^{3}$ However, later reports using more standardised criteria showed significantly lesser incidence and the cause of IBC still remained elusive. ${ }^{4}$
After separate guidelines for the work-up and treatment of patients with IBC were finally developed and published in 2008, fewer patients with IBC are misdiagnosed for $\angle A B C$ because major clinical and pathological characteristics have become widely known. ${ }^{5}$ Today it is well established that IBC represents a completely distinct clinical entity, different from $\angle A B C$, with different clinical and pathology presentation and molecular characteristics, as well as treatment outcome and prognosis. ${ }^{6}$

The clinical presentation of IBC is usually impressive, with rapidly developed skin redness, oedema and breast enlargement (see Figure 2 and 3). The other prominent characteristic of IBC is frequent absence of underlying $B C$; in fact this is the only form of $B C$ that could be diagnosed actually without detectable, formed invasive breast carcinoma. ${ }^{6}$ Approximately $50 \%$ of IBC patients do not have palpable mass, or mammogram evidence of underlying $\mathrm{BC} .{ }^{7.8}$ Of course, it is assumed that histological proof of the underlying malignant nature of mastitis must always be obtained. However, when underlying BC is present, all histological types have been identified. ${ }^{9-11}$ Most frequently it is ductal and lobular carcinoma, with a minimal or absent in situ component as a prominent growth pattern of $\mathrm{IBC}$; therefore, no recognised precursor lesions for $\mathrm{IBC}$ have been identified. ${ }^{12}$ Major molecular characteristics are also defined and it is known that up to $83 \%$ of IBC tumours lack oestrogen receptor (ER) expression, while HER2 overexpression is significantly higher than in non-IBC patients. ${ }^{13-18}$ However, an International IBC Panelist recently stated that the true prognostic significance of HER2 overexpression among women with $\mathrm{IBC}$ is currently still unknown, in contrast to the well-known negative prognostic impact that HER2 overexpression has in common BC. ${ }^{19-22}$ However, insufficiently explored prognostic significance does not 
interfere with HER2 expression's well-known predictive significance to trastuzumab treatment, and all IBC patients with HER2 overexpression should be treated with trastuzumab.

\section{Impact of Dermal Lymphatic Invasion on the Prognosis of Inflammatory Breast Carcinoma}

Historically, clinical presentation that resembles non-malignant acute mastitis was the main reason for delay in diagnosis and treatment in many patients. However, this typical clinical presentation of breast skin inflammation is not due to true physiological inflammatory response but is rather a consequence of pathological plugging of the dermal lymphatic of the breast with tumour emboli.

This pathological phenomenon was observed long ago, when Thomas Bryant in 1887 observed pathological tumour invasion of the dermal lymphatic vessels, recognising that lymphatic obstruction produced the clinical appearance of inflammation. ${ }^{23}$

Later, IBC was determined as a clinical entity in which confirmation of dermal lymphatic invasion (DLI) is equivalent to 'pathological proof' of this rare malignancy, because it is caused by filling of the dilated dermal Iymphatics by tumour emboli, subsequently leading to lymphatic obstruction and producing the typical inflammatory appearance..$^{24}$

This was so convincing as a dominant pathology event for many researchers that they preferred the pathological over the clinical definition for IBC, proposing "dermal lymphatic carcinomatosis of the breast" as the alternative phrase instead of inflammatory breast carcinoma. ${ }^{25,26}$

However, it was soon recognised that even with an adequate number of tissue-block samples and multiple sections $(\geq 10)$, dermal lymphatic tumour emboli are evident in up to $80 \%$ of patients with true primary IBC and the diagnosis of inflammatory carcinoma is still made primarily on clinical grounds. However, the absence of DLI does not exclude the diagnosis of IBC. ${ }^{5}$

\section{Inflammatory Breast Carcinoma Subtypes}

Because of those peculiar characteristics, it has been suggested that there may be three subtypes of IBC according to combined clinical and/or pathological features. ${ }^{27-31}$

The first IBC subtype is characterised by clinical inflammation but without pathological plugging of the dermal lymphatic. The second subtype has pathological plugging of the dermal lymphatic but without clinical signs of inflammation. And finally, the third subtype is fully developed IBC with both clinical inflammation and pathological involvement of the dermal lymphatic. It could be speculated that prognosis of patients with the above-mentioned three types of IBC differs, and that a combination of DLI with a full inflammatory component might be the worst. Among all mentioned IBC subtypes, the rarest is one with DLI but without an inflammatory component, and from a clinical aspect the true prognostic significance of this rare IBC subtype is still a matter of debate. According to the results of one study, 836 mastectomy specimens were re-examined and only 15 patients (1.8\%) were identified with DLI but without inflammatory disease (ID). ${ }^{31}$ In another study, with an impressively high number of breast cancer specimens examined $(51,030)$, a total of 3,172 patients had IBC (6\%). ' Among these patients only 81 had DLI but without the inflammatory component of $\mathrm{IBC}$, which represents only $0.16 \%$ of
Figure 1: Locally Advanced Breast Carcinoma with Secondary Inflammation

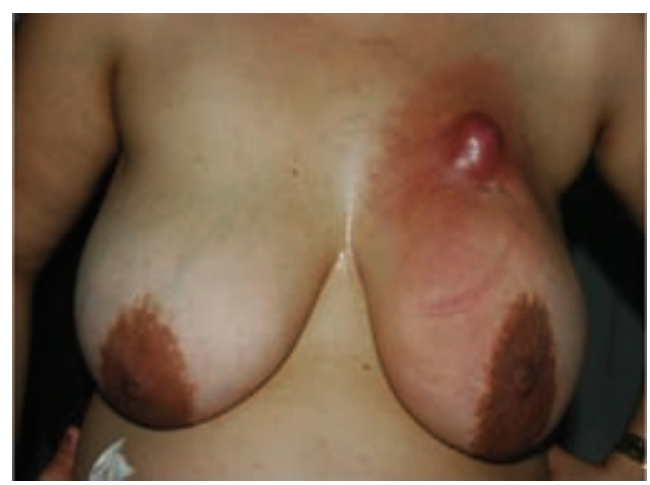

Figure 2: Inflammatory Breast Carcinoma

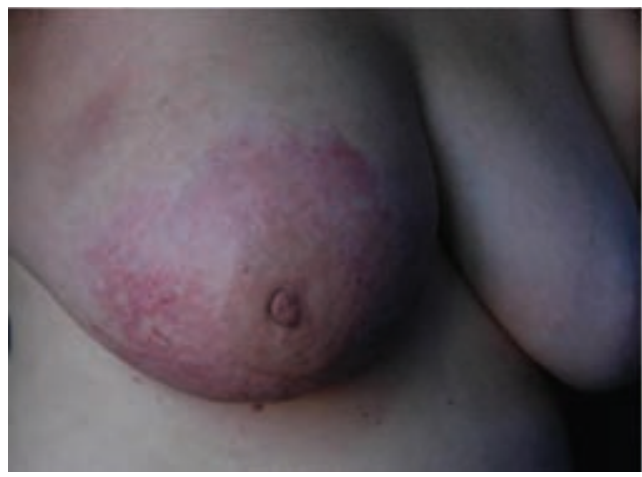

Figure 3: Inflammatory Breast Carcinoma

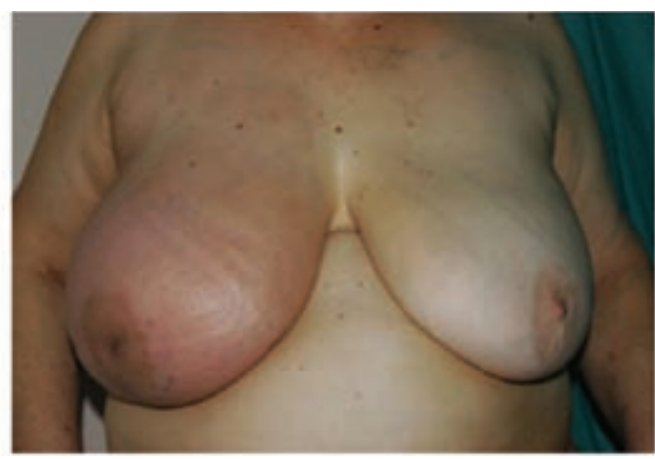

patients with IBC. Therefore, this subtype clearly represents the rarest form of IBC.

However, skin involvement in IBC is technically always interpreted as a metastatic site at the time of primary diagnosis. Therefore, it could be expected that proven skin involvement actually represents the most important factor for generally poorer prognosis of patients with IBC. In fact, it has been proposed that DLI is responsible for the high metastatic potential of IBC. . $^{32}$

Indeed, the impact of DLI on clinical course and definitive outcome has been explored, but in a surprisingly small number of publications. Because contemporary anthracycline- and taxane-based chemotherapy treatment is now widely used in everyday clinical practice, the prognosis of patients with IBC has been generally improved. There are reports of long-term survival, with five-year 
survival exceeding $40 \%$ compared with the historical controls with only $5 \%$ INC patients surviving that period. Moreover, even 15-year survival in $\sim 20 \%$ of patients was reported. ${ }^{8,19}$ However, to the best of our knowledge, these studies have not explored whether there are differences in treatment outcomes according to the presence or absence of DLI in IBC.

In the literature review, we have found only a few studies specifically addressing the significance of DLI in IBC prognosis.

In one small study authors explored whether tumour emboli in dermal lymphatics without inflammatory signs represent a similar bad prognosis like with classical inflammatory breast cancer. ${ }^{33}$

They evaluated the prognostic role of DLI in breast cancer with inflammatory disease (DLI + ID) or without (DLI W/O ID) ID. Forty-two patients were identified with DLI. Twenty-five were classified as PT4, 13 of them as pT4d (inflammatory disease); the 17 remaining patients had cancers with DLI. The American Joint Committee on Cancer (AJCC) staging system was used ${ }^{34}$ After a mean follow-up of 33 months, 22/42 patients (52 \%) were disease free. The three-year disease-free survival (DFS) was 50 \% (DLI W/O ID, $61 \%$; DLI + ID, 31 \%; $\mathrm{p}<0.03$ ); the corresponding overall survival (OS) was $69 \%$ (DLI W/0 $I D, 87 \% ; D L I+I D, 37 \% ; p=0.005)$. All patients were treated with various chemo and/or hormonal treatments, as well as surgery and/or radiotherapy.

In that small study, the presence or absence of ID was the only significant parameter for all end-points in multivariate analyses. Despite the same histopathologic presentation, DLI without ID was accompanied by significantly better DFS and OS than ID.

In a larger study, the outcome of 163 IBC patients with or without DL was compared with the outcome of 99 patients with proven DLI but without an inflammatory component. ${ }^{35}$ Both five-year DFS and OS were significantly worse in patients with an inflammatory component (five-year DFS $25.6 \%$ versus $51.6 \%$; $p=0.0001$; OS $28.6 \%$ versus $40 \%$; $\mathrm{p}=0.05$ ). Authors also explored all relevant patients and molecular characteristics, such as age, nodal status, hormonal receptors status, and found no multivariate statistical significant differences between DLI with or without an inflammatory component.

In a recently published study, 432 patients with $\mathrm{BC}$ underwent post-mastectomy irradiation. ${ }^{36}$
The authors addressed the influence of DLI with or without an inflammatory component on definitive patient's outcome. They identified 31 patients with IBC without DLI, 21 patients with DLI but without an inflammatory component and 18 patients with both DLI and an inflammatory component. The main conclusion is that DLI and IBC are independent predictors of poor outcome, but also that patients with both characteristics have a worse prognosis with increased risk for local and distant relapse.

Similar findings were presented in a study published in 1995, ${ }^{28}$ with the main conclusion that inflammatory presentation alone, without either DLI or extensive lymph nodal involvement, heralds better outcome.

At the Institute for Oncology and Radiology of Serbia, we have explored whether the presence of DLI in IBC influences chemotherapy response, time to progression and OS. Eighty-five patients with IBC stage III were identified between January 2008 and March 2010. Forty patients (47 \%) had confirmed DLI and 45 patients (53\%) did not have confirmed DLI by multiple-site skin biopsy. All patients were treated with induction anthracycline-based chemotherapy for four-six cycles, and treatment response was evaluated by mammography and clinically. Less complete response (CR) and partial response (PR) were registered in the subgroup of patients with DLI (55\% versus $75 \%$ ). Also, time to progression (TTP) was shorter in patients with DLI (TTP 12.8 months versus 14.8 months) and OS was also poorer (12.5 months versus 20.0 months). ${ }^{37}$

\section{Conclusion}

Whether DLI presence could represent a real negative factor in respect to treatment outcome should be explored in prospective clinical studies, in correlation with all significant molecular parameters and types of systemic treatment. Until results from such studies become available, current data should be interpreted with caution as results are obtained from small patient populations.

Individual patient prognosis with IBC still cannot be predicted, except that better outcome could be expected in those who obtain pathological complete response after chemotherapy. ${ }^{19}$

For most patients, the statement from previous studies that IBC could be defined either clinically or pathologically, but the prognosis would be poor either way, still stands. ${ }^{28,38}$
1. Levine PH, Steinhorn SC, Ries LG, et al., Inflammatory breast cancer: The experience of the Surveillance, Epidemiology, and End Results (SEER) program, I Natl Cancer Inst, 1985:74:291-7.

2. Lee $B$, Tannenbaum $E$, Inflammatory carcinoma of the breast, Surg Gynecol Obstet, 1924;39:580-95.

3. Mourali N, Muenz LR, Tabbane F, et al., Epidemiologic features of rapidly progressing breast cancer in Tunisia Cancer, 1980;46(12):2741-6.

4. Boussen $\mathrm{H}$, Bouzaiene $\mathrm{H}$, Farouk B, et al., Inflammatory breast cancer in Tunisia: epidemiological and clinical trends, Cancer, 2010;116:2730-5.

5. Dawood S, Broglio K, Gong Y, et al., Prognostic significance of HER-2 status in women with inflammatory breast cancer, Of HER-2 status in women

6. Breast, In: Green F, Page D, Fleming I, et al., eds, AJCC Cancer Staging Manual, 6th edn, New York: Springer-Verlag, 2002, 225-81.

7. Kushwaha AC, Whitman GJ, Stelling CB, et al., Primary inflammatory carcinoma of the breast: retrospective review of mammographic findings, AJR Am I Roentgenol, 2000;174(2);535-8.

8. Ueno NT, Buzdar AU, Singletary SE, et al., Combined-modality treatment of inflammatory breast carcinoma: twenty years of experience at M. D. Anderson Cancer Center, Cancer Chemother Pharmacol, 1997:40(4):321-9.

9. Jaiyesimi IA, Buzdar AU, Hortobagyi G, et al., Inflammatory breast cancer: a review, J Clin Oncol, 1992;10:1014-2

10. Giordano SH, Hortobagyi GN, Inflammatory breast cancer: clinical progress and the main problems that must be addressed, Breast Cancer Res, 2003;5(6):284-8

11. Harris EE, Schultz D, Bertsch H, et al., Ten-year outcome after combined modality therapy for inflammatory breas cancer, Int J Radiat Oncol Biol Phys, 2003;55(5):1200-8.

12. Anderson WF, Schairera C, Chena BE, et al., Epidemiology of Inflammatory Breast Cancer (IBC), Breast Dis, 2005;22:9-23.

13. Sawaki M, Ito $Y$, Akiyama F, et al., High prevalence of HER$2 /$ neu and p53 overexpression in inflammatory breast 2/neu and p53 overexpression in in

14. Guerin $M$, Gabillot M, Mathieu MC, et al., Structure and expression of c-erbB-2 and EGF receptor genes in inflammatory and non-inflammatory breast cancer: prognostic significance, Int J Cancer, 1989;43:201-8.

15. Rosen PP, Inflammatory carcinoma, In: Rosen PP, ed., Rosen's Breast Pathology (edn 2), Philadelphia: Lippincott Williams \& Wilkins, 2001, 676-83.

16. Harvey HA, Lipton A, Lawrence BV, et al., Estrogen receptor status in inflammatory breast carcinoma, I surg Oncol,
1982;21:42-4

17. Charpin C, Bonnier P, Khouzami A, et al., Inflammatory breast carcinoma: an immunohistochemical study using monoclonal anti-pHER-2/neu, pS2, cathepsin, ER and PR, Anticancer Res, 1992;12:591-7.

18. Guerin M, Sheng ZM, Andrieu N, Riou G, Strong association between c-myb and oestrogen-receptor expression in human breast cancer, Oncogene, 1990;5:131-5.

19. Dawood S, Merajver D, Viens P, et al., International expert panel on inflammatory breast cancer: consensus statement for standardized diagnosis and treatment, Annals oncol, 2011;22:515-23.

20. Zell JA, Tsang WY, Taylor TH, et al., Prognostic impact of human epidermal growth factor-like receptor 2 and hormone receptor status in inflammatory breast cancer (IBC): analysis receptor status in inflammatory breast cancer (IBC): ana
of 2,014 IBC patient cases from the California Cancer Registry, Breast Cancer Res, 2009;11:R9.

21. Hance KW, Anderson WF, Devesa SS, et al., Trends in inflammatory breast carcinoma incidence and survival: the surveillance, epidemiology, and end results program at the National Cancer Institute, I Natl Cancer Inst, 2005;97:966-75.

22. Parton M, Dowsett $M$, Ashley $S$, et al., High incidence of HER2 positivity in inflammatory breast cancer, Breast, 2004:13:97-103. 
23. Bryant, T, Diseases of the Breast, London, Paris, New York Melbourne: Cassell \& Company, 1887,186-94

24. Taylor GW, Meltzer A, "Inflammatory carcinoma" of the breast, Am I Cancer, 1938;33:33-49.

25. Saltzstein SL, Clinically occult inflammatory carcinoma of the breast, Cancer, 1974;34:382-8.

26. Ellis $\mathrm{DL}$, Teitelbaum $\mathrm{SL}$, Inflammatory carcinoma of the breast. A pathologic definition, Cancer, 1974:33:1045-7.

27. Amparo RS, Angel $C D$, Ana $L H$, et al., Inflammatory breast carcinoma: pathological or clinical entity? Breast Cancer Res Treat, 2000;64:269-73

28. Bonnier P, Charpin C, Lejeune C, et al., Inflammatory carcinomas of the breast: a clinical, pathological, or a clinica and pathological definition? Int / Cancer, 1995;62:382-5
29. Bonnier P, Piana L, Khouzami A, et al., Inflammatory carcinoma of the breast, Eur I Gynaecol Oncol, 1992;13:7-11.

30. Levine $\mathrm{PH}$, Veneroso $\mathrm{C}$, The epidemiology of inflammatory breast cancer, Semin Oncol, 2008;35:11-6.

31. Lucas FV, Perez-Mesa C, Inflammatory carcinoma of the breast, Cancer, 1978;41:1595-605. 32. Jardines $L$, Haffty B, Theriault R, Locally advanced locally recurrent and metastatic breast cancer, In: Pazdur R, Coia $L$,
Hoskins $W$, wagman $L$, eds, Cancer Management. A Hoskins W, Wagman L, eds, Cancer Management. A Multidisciplinary Approach, 3rd edn, Melville: PRR, 1999, 73-88.

33. Gruber G, Ciriolo M, Altermatt HJ, et al., Prognosis of derma Iymphatic invasion with or without clinical signs of inflammatory breast cancer, Int I Cancer, 2004;109(1):144-8. 34. Singletary SE, Connolly JL, Breast cancer staging: working with the sixth edition of the AJCC cancer staging manual, $C A$ Cancer I Clin, 2006;56:37-47

35. Amparo RS, Angel $C D$, Ana $L H$, et al., Inflammatory breast carcinoma: pathological or clinical entity? Breast Cancer Res Treat, 2000;64:269-73

36. Abramowitz MC, Li T, Morrow M, Sigurdson ER, et al., Derma lymphatic invasion and inflammatory breast cancer are independent predictors of outcome after post mastectomy radiation, Am J Clin Oncol, 2009;32(1):30-3.

37. Kolarevic $D$, Tomasevic Z, Inflammatory Breast Cancer: Does the confirmation of dermal lymphatic invasion (DLI) predict the worse outcome? EJC, 2011;47:S353.

38. Kuske RR, Diagnosis and management of inflammatory -breast cancer, Semin Radiat oncol, 1994:4:270-82. 